Pure and Applied Mathematics Quarterly

Volume 2, Number 4

(Special Issue: In honor of

Robert MacPherson, Part 2 of 3)

1053-1084, 2006

\title{
Stratified Morse Theory: Past and Present
}

\author{
David B. Massey \\ In honor of Robert MacPherson on his 60th birthday
}

\begin{abstract}
We recall the basic definitions and results of Goresky and MacPherson on Stratified Morse Theory. We also briefly describe the "classical" applications of these results to intersection homology, homotopy results for algebraic varieties, and homology groups of complements of affine subspace arrangements. We then discuss a number of related results and developments, which have appeared since Goresky and MacPherson's work.
\end{abstract}

\section{INTRODUCTION}

In 1974, Mark Goresky and Robert MacPherson began their development of intersection homology theory, and their first paper on this topic appeared in 1980; see [12]. At that time, they were missing a fundamental tool which was available for the study of smooth manifolds; they had no Morse Theory for stratified spaces. Goresky and MacPherson wished to have a Stratified Morse Theory to allow them to prove a Lefschetz hyperplane theorem for the intersection homology of complex singular spaces, just as ordinary Morse Theory yields the Lefschetz Hyperplane Theorem for ordinary homology of complex manifolds ([34], §7).

The time was ripe for a stratified version of Morse Theory. In 1970, Mather had given a rigorous proof of Thom's first isotopy lemma [33]; this result says that proper, stratified, submersions are locally-trivial fibrations. In 1973, Morse functions on singular spaces had been defined by Lazzeri in [25], and the density and stability of Morse functions under perturbations had been proved in [37]. We shall recall these definitions and results in Section 2.

What was missing was the analog of the fundamental result of Morse Theory, a theorem describing how the topology of a space is related to the critical points of a proper Morse function. In [16], Goresky and MacPherson proved such a theorem

Received January 29, 2006. 
for stratified spaces. Suppose that $M$ is a smooth manifold, that $X$ is a Whitney stratified subset of $M$, and that $f: X \rightarrow \mathbb{R}$ is a proper function which is the restriction of a smooth function on $M$. For all $v \in \mathbb{R}$, let $X_{\leq v}:=f^{-1}((-\infty, v])$. Suppose that $a, b \in \mathbb{R}, a<b$, and $f^{-1}([a, b])$ contains a single (stratified) critical point, $p$, which is non-degenerate (see Definition 2.3) and contained in the open set $f^{-1}((a, b))$. Let $S$ be the stratum containing $p$. Then, the Main Theorem of Stratified Morse Theory (see Theorem 2.16) says that the topological space $X_{\leq b}$ is obtained from the space $X_{\leq a}$ by attaching a space $A$ to $X_{\leq a}$ along a subspace $B \subseteq A$, where the pair $(A, B)$, the Morse data, is the product of the tangential Morse data of $f$ at $p$ and the normal Morse data of $f$ at $p$. This result is especially powerful in the complex analytic case, where the normal Morse data depends on the stratum $S$, but not on the point $p$ or on the particular Morse function $f$. Detailed proofs of these results appeared in the 1988 book Stratified Morse Theory [16]; we present a summary of a number of these results in Section 2.

Even before the appearance of [16], Goresky and MacPherson published two papers, Stratified Morse Theory [15] and Morse Theory and Intersection Homology Theory [14], which contained announcements of many of the fundamental definitions and results of Stratified Morse Theory. In addition, these two papers showed that Stratified Morse Theory has a number of important applications to complex analytic spaces, including homotopy results, the desired Lefschetz Hyperplane Theorem for intersection homology, and the first proof that the (shifted) nearby cycles of a perverse sheaf are again perverse. We shall discuss these results and others in Section 3.

While it is probably fair to say that most papers on the topology of singular spaces which have appeared in the last 15 years use Stratified Morse Theory as a tool at some point, many of these papers are not really about Stratified Morse Theory. In Section 4, we will discuss results which has appeared since the publication of [16] which are, in fact, closely related to Stratified Morse Theory. In these works, either Stratified Morse Theory is the fundamental tool, or the results are improvements/refinements of results found in [16], or the results are variations of the Main Theorem that local Morse data is the product of tangential and normal Morse data. We also include a very brief discussion of discrete Morse Theory.

We should remark that this paper is not a complete treatment of Stratified Morse Theory. For such a treatment, the reader is directed to [16]; the exposition and pictures provided by Goresky and MacPherson are outstandingly beautiful, especially for such a technical subject. 


\section{Basic Stratified Morse Theory}

In this section, we will briefly present the fundamental definitions and results from [16], [15], and [14]; we will discuss the applications of these results in the next section.

\section{STRATIFICATIONS AND DECOMPOSITIONS}

Fix a smooth $\left(C^{\infty}\right)$ manifold $M$, and a subset $X \subseteq M$ with a Whitney stratification $\mathfrak{S}$ (where we are not assuming that the strata are connected). For $S_{\alpha}, S_{\beta} \in \mathfrak{S}$, we write $S_{\alpha} \leq S_{\beta}$ for the usual ordering given by $S_{\alpha} \subseteq \overline{S_{\beta}}$. For $x \in X$, let $S_{x}$ denote the stratum of $\mathfrak{S}$ containing $x$.

Many of the results and constructions in Stratified Morse Theory refer to topological spaces which can be written as disjoint unions of subspaces which are naturally indexed by the stratification $\mathfrak{S}$, even though the spaces themselves are not stratified by $\mathfrak{S}$. For instance, this is the case in the Main Theorem of Stratified Morse Theory (Theorem 2.16). Thus, one is led to define the notion of a decomposition. One can define decompositions with respect to any partially-ordered set; however, for our purposes, it suffices to consider the partially-ordered set $\mathfrak{S}$.

Definition 2.1.([16], §I.1.1) An S-decomposition of a topological space $Z$ is a locally finite collection $\left\{Z_{S} \mid S \in \mathfrak{S}\right\}$ of disjoint, locally closed, subsets of $Z$ such that $Z=\bigcup_{S \in \mathfrak{S}} Z_{S}$ and

$$
Z_{S_{\alpha}} \cap \overline{Z_{S_{\beta}}} \neq \emptyset \Longleftrightarrow Z_{S_{\alpha}} \subseteq \overline{Z_{S_{\beta}}} \Longleftrightarrow S_{\alpha} \leq S_{\beta}
$$

Suppose that $Y$ is a subspace of $X$, and $P$ is a topological space. Then, $Y$ and $P \times Y$ have canonical $\mathfrak{S}$-decompositions; if $S \in \mathfrak{S}$, then we let $Y_{S}:=S \cap Y$ and $(P \times Y)_{S}:=P \times Y_{S}$, and define decompositions $\mathfrak{S}_{Y}:=\left\{Y_{S} \mid S \in \mathfrak{S}\right\}$ and $\mathfrak{S}_{P \times Y}:=\left\{(P \times Y)_{S} \mid S \in \mathfrak{S}\right\}$.

An $\mathfrak{S}$-decomposed map $g$ between two $\mathfrak{S}$-decomposed spaces $W$ and $Z$ is a continuous function which "preserves strata", i.e., for all $S \in \mathfrak{S}, g\left(W_{S}\right) \subseteq Z_{S}$. If $A, B$, and $Z$ are $\mathfrak{S}$-decomposed spaces, where $B \subseteq A$, and $g: B \rightarrow Z$ is an $\mathfrak{S}$ decomposed map, then the adjunction space $Z \cup_{B} A$ is canonically $\mathfrak{S}$-decomposed.

If two $\mathfrak{S}$-decomposed spaces are homeomorphic via an $\mathfrak{S}$-decomposed homeomorphism, then we say that the spaces are $\mathfrak{S}$-homeomorphic.

Note that these decompositions of $Y$ and $Y \times P$ can easily fail to be Whitney stratifications. However, if $N$ is a smooth submanifold of $M$ which transversely intersects all of the strata of $\mathfrak{S}$, then the $\mathfrak{S}$-decomposition of $N \cap X$ is, in fact, a Whitney stratification. 
Assume now that $M$ is endowed with a Riemannian metric; all of the results that we discuss are independent of the choice of this metric. We let $B_{\delta}(x)$ denote the closed ball of radius $\delta$ centered at $x \in M$. In the following definition, we follow [16], §I.1.4; however, we have altered the terminology slightly, in an attempt to more accurately match current usage.

Definition 2.2. If $x \in X$, then, there exists $\delta_{0}>0$ such that, for all $\delta$ such that $0<\delta \leq \delta_{0}$, the boundary sphere $\partial B_{\delta}(x)$ is transverse to the strata of $\mathfrak{S}$, and so the $\mathfrak{S}$-decomposition of $\partial B_{\delta}(x) \cap X$ is actually a Whitney stratification; thus, $B_{\delta}(x) \cap X$ has a canonical Whitney stratification, which is a refinement of its S-decomposition. We refer to such a $B_{\delta}(x)$ as a Whitney ball at $x$ in $M$, and the S-decomposed Whitney stratified space $B_{\delta}(x) \cap X$ as a Whitney ball at $x$ in $X$. The $\mathfrak{S}$-decomposed Whitney stratified space $\partial B_{\delta}(x) \cap X$ is the real link of $X$ at $x$. The $\mathfrak{S}$-decomposed Whitney stratified homeomorphism-type of the pair $\left(B_{\delta}(x) \cap X, \partial B_{\delta}(x) \cap X\right)$ is independent of the Riemannian metric, the Whitney ball, and the choice of $x$ in a single connected component of the stratum $S_{x}$.

Let $x \in X$, and let $N$ be a smooth submanifold of $M$ which transversely intersects all of the strata of $\mathfrak{S}$, and such that $N \cap S_{x}=\{x\}$. Then, $N$ is a normal submanifold to $X$ at $p$, and the $\mathfrak{S}$-decomposed Whitney stratified space $N \cap X$ is called a normal slice of $X$ at $x$. A normal ball to $X$ at $x$ is a Whitney ball at $x$ in $N \cap X$.

The real link of the stratum $S_{x}$ at $x$ is the is the real link of the stratum $\{x\}$ in $N \cap X$. The $\mathfrak{S}$-decomposed Whitney stratified homeomorphism-type of the pair $\left(N \cap X \cap B_{\delta}(x), N \cap X \cap \partial B_{\delta}(x)\right)$ is independent of the choice of $N$, the Riemannian metric, the Whitney ball, and the choice of $x$ in a single connected component of the stratum $S_{x}$.

The reader should note that the term "normal slice" is frequently used to mean any one of the three spaces: $N \cap X \cap B_{\delta}(x)$ (as in [16]), $N \cap X$, or $N$; however, the meaning is always clear from the context.

If $B_{\delta}(x)$ is a Whitney ball, and we write $\operatorname{cone}\left(\partial B_{\delta}(x)\right)$ for

$$
\left(\partial B_{\delta}(x) \times[0,1]\right) /\left(\partial B_{\delta}(x) \times\{0\}\right),
$$

then there exists a homeomorphism $h: B_{\delta}(x) \rightarrow \operatorname{cone}\left(\partial B_{\delta}(x)\right)$ such that $h(x)=$ cone point, and the restriction of $h$ to a map from $B_{\delta}(x)-\{x\}$ to $\partial B_{\delta}(x) \times(0,1]$ is $\mathfrak{S}$-decomposed.

\section{COTANGENTS AND CONORMALS}

Fix a smooth function $\tilde{f}: M \rightarrow \mathbb{R}$, and let $f:=\tilde{f}_{\left.\right|_{X}}$. Such an $f$ is simply referred to as a smooth function on $X$. The (stratified) critical locus of $f$ is 
$\Sigma_{\mathfrak{S}} f:=\bigcup_{\alpha} \Sigma\left(f_{\left.\right|_{S_{\alpha}}}\right)$, where $\Sigma\left(f_{\left.\right|_{S_{\alpha}}}\right)$ is the ordinary critical locus of a function on a smooth manifold.

Let us recall some notions from conormal geometry. For $S_{\alpha} \in \mathfrak{S}$, we let $T_{S_{\alpha}}^{*} M$ denote the conormal space to $S_{\alpha}$ in $M$, i.e., $T_{S_{\alpha}}^{*} M$ is a vector bundle over $S_{\alpha}$, whose fiber $\left(T_{S_{\alpha}}^{*} M\right)_{x}$ over a point $x \in S_{\alpha}$ consists of the cotangent vectors (covectors) in $\left(T^{*} M\right)_{x}$ which vanish on the tangent space to $S_{\alpha}$ at $x$. By Whitney's condition A, $T_{\mathfrak{S}}^{*} M:=\bigcup_{\alpha \in A} T_{S_{\alpha}}^{*} M$ is a closed subset of $\left(T^{*} M\right)_{\left.\right|_{X}}$; the elements of $T_{X}^{*} M$ are called conormal (or, characteristic) covectors of $X$ (in $T^{*} M$ with respect to $\mathfrak{S})$. If the stratification of $X$ is clear, we usually write $T_{X}^{*} M$ in place of $T_{\mathfrak{S}}^{*} M$. Note that $x \in \Sigma_{\mathfrak{S}} f$ if and only if $d_{x} \tilde{f} \in T_{S_{x}}^{*} M$.

The set of degenerate conormal covectors to a stratum $S_{\alpha}$ is defined to be

$$
D_{S_{\alpha}}^{*} M:=T_{S_{\alpha}}^{*} M \cap \bigcup_{S_{\alpha}<S_{\beta}} \overline{T_{S_{\beta}}^{*} M}=\left(\bigcup_{S_{\alpha}<S_{\beta}} \overline{T_{S_{\beta}}^{*} M}\right)_{\left.\right|_{S_{\alpha}}} .
$$

Thus, the fiber $\left(D_{S_{x}}^{*} M\right)_{x}$ consists of limits at $x$ of conormal covectors to larger strata or, equivalently, conormal covectors to $S_{x}$ at $x$ which vanish on limiting tangent spaces from larger strata.

Suppose that we have another smooth manifold $N$ and a smooth map $g$ : $M \rightarrow N$. Then, a covector $\xi \in T_{q} N$ is characteristic (for $g$ and $\mathfrak{S}$ ) if there exists a $p \in X \cap g^{-1}(q)$ such that the pullback $g^{*}(\xi):=\xi \circ d_{p} g \in T_{p}^{*} M$ is a characteristic covector of $X$.

\section{MORSE FUNCTIONS AND NONDEPRAVED CRITICAL POINTS}

Now we are ready to define Morse functions in the stratified situation.

Definition 2.3.([25], [37]) A point $x \in X$ is a (stratified) nondegenerate critical point of $f$ if and only if $x$ is a nondegenerate critical point of $f_{\left.\right|_{S_{x}}}$ and $d_{x} \tilde{f} \notin D_{S_{x}}^{*} M$.

The function $f$ is a Morse function if and only if $f$ is proper, has distinct critical values, and all of the critical points of $f$ are nondegenerate.

Note that a nondegenerate critical point of $f$ is necessarily an isolated point in $\Sigma_{\mathfrak{S}} f$.

It follows from the work of Pignoni [37] and others (see I.2.2.1 of [16]) that if $M$ is an analytic manifold, $X$ is a closed subanalytic set of $M$, and $\mathfrak{S}$ is a subanalytic Whitney stratification, then:

Theorem 2.4. The set of smooth, proper functions from $M$ into $\mathbb{R}$, which restrict to Morse functions on $X$, form an open, dense subset (in the Whitney $C^{\infty}$ topology) of the set of all smooth, proper functions from $M$ into $\mathbb{R}$. 
Thus, any smooth, proper function on $M$ can be perturbed slightly to produce a function which restricts to a Morse function on $X$.

Another familiar "genericity" result from ordinary Morse theory generalizes to Stratified Morse Theory:

Theorem 2.5.([16], I.2.2.4) Suppose that $M=\mathbb{R}^{n}$. For $p \in M$, let $r_{p}: M \rightarrow \mathbb{R}$ denote the distance squared function (from $p$ ). Then, for almost all $p \in M,\left(r_{p}\right)_{\left.\right|_{X}}$ is a Morse function.

Essentially, Stratified Morse Theory is the theory which describes what a Morse function on a stratified space tells one about the topology of the space. However, the Main Theorem of Stratified Morse Theory (Theorem 2.16) applies to critical points which are more general than nondegenerate critical points; the theorem applies to what Goresky and MacPherson termed as nondepraved critical points. This more general notion is useful since it includes, for instance, isolated critical points of real analytic functions, even if the critical point is degenerate.

Definition 2.6. $([16], \S I .2 .3)$ Let $\mathcal{U}$ be an open subset of $\mathbb{R}^{n}$, and let $g: \mathcal{U} \rightarrow \mathbb{R}$ be a smooth function. Then, an isolated critical point $p$ of $g$ is nondepraved if and only if it satisfies the following condition: Suppose that $p_{i}$ is a sequence of points in $\mathcal{U}$, and that the vectors $v_{i}:=\left(p_{i}-p\right) /\left|p_{i}-p\right|$ converge to some limiting vector $v$. Suppose that the hyperplanes ker $d_{p_{i}} g$ converge to some limiting hyperplane $\tau$, and that $v \notin \tau$. Then, for all sufficiently large $i, d_{p_{i}} g\left(v_{i}\right) \cdot\left(g\left(p_{i}\right)-g(p)\right)>0$.

In order to define a nondepraved critical point of a function on a smooth manifold, we need to know that nondepravity is invariant under diffeomorphisms.

Proposition 2.7. $([16], \S I .2 .6)$ Suppose that $\mathcal{U}$ and $\mathcal{V}$ are open neighborhoods of the origin in $\mathbb{R}^{n}$, and that $\phi:(\mathcal{U}, \mathbf{0}) \rightarrow(\mathcal{V}, \mathbf{0})$ is a diffeomorphism. Then, $\mathbf{0}$ is a nondepraved critical point of a smooth function $g: \mathcal{V} \rightarrow \mathbb{R}$ if and only if $\mathbf{0}$ is a nondepraved critical point of $g \circ \phi$.

Definition 2.8. If $N$ is a smooth manifold and $g: N \rightarrow \mathbb{R}$ is smooth, then $p \in N$ is a nondepraved critical point of $g$ if and only if, for some smooth coordinate patch $\phi$ on $N$ at $p, \phi(p)$ is a nondepraved critical point of $g \circ \phi^{-1}$.

Proposition 2.9. If $p$ is a nondegenerate critical point of a smooth function $g$ on a smooth manifold, then $p$ is nondepraved.

Proof. By the Morse Lemma and Proposition 2.7, one is reduced to showing that the origin is a nondepraved critical point of $g=-x_{1}^{2}-\cdots-x_{\lambda}^{2}+x_{\lambda+1}^{2}+\ldots x_{n}^{2}$. This is trivial. 
Proposition 2.10.([16], §I.2.4) If $N$ is a real analytic manifold, and $g: N \rightarrow$ $\mathbb{R}$ is a real analytic function, with an isolated critical point at $p$, then $p$ is a nondepraved critical point of $g$.

Now, we return to the stratified setting.

Definition 2.11.([16], §I.2.3) A critical point $x \in X$ of the function $f: X \rightarrow \mathbb{R}$ is nondepraved (with respect to $\mathfrak{S}$ ) if and only if $f_{\left.\right|_{S x}}$ has a nondepraved critical point at $x$ and $d_{x} \tilde{f} \notin D_{S_{x}}^{*} M$.

Note that a nondepraved critical point of $f$ is necessarily an isolated point in $\Sigma_{\mathfrak{S}} f$.

\section{MORSE DATA}

Throughout this subsection on Morse data, we assume that our smooth function $f: X \rightarrow \mathbb{R}$ is proper, and that $p \in X$ is a nondepraved critical point of $f$. Let $v:=f(p)$.

In the remainder of this section, there will be a number of $\mathfrak{S}$-decomposed and/or stratified spaces which are defined after making a choice of real numbers $\delta$ and $\epsilon$ such that $0<\epsilon \ll \delta \ll 1$. When we do this, we mean that this characterizes a unique $\mathfrak{S}$-decomposed and/or stratified homeomorphism-type. When we write that the space is independent of the choice of $0<\epsilon \ll \delta \ll 1$, we actually mean that this homeomorphism-type is independent of the pair $(\epsilon, \delta)$ in some fringed set; see [16], Chapter 5.

For $a, b \in \mathbb{R}$, let $X_{a}:=f^{-1}(a), X_{\leq a}:=f^{-1}((-\infty, a])$, and $X_{[a, b]}:=f^{-1}([a, b])$. Recall that $S_{p}$ denotes the stratum containing $p$.

If $p$ is the only critical point of $f$ with critical value $v$, then it follows easily that there exists $\epsilon_{0}>0$ such that $\left[v-\epsilon_{0}, v+\epsilon_{0}\right]$ contains no critical values of $f$ other than $v$. By Thom's first isotopy lemma, for $0<\epsilon \leq \epsilon_{0}, Z_{v-\epsilon_{0}}$ (respectively, $Z_{v+\epsilon_{0}}$, resp., $\left.Z_{\left[v-\epsilon_{0}, v+\epsilon_{0}\right]}\right)$ is homeomorphic to $Z_{v-\epsilon}$ (respectively, $Z_{v+\epsilon}$, resp., $Z_{[v-\epsilon, v+\epsilon]}$ ) by an S-decomposed homeomorphism; note that the S-decompositions of $Z_{v-\epsilon}$ and $Z_{v+\epsilon}$ are Whitney stratifications, and that $Z_{[v-\epsilon, v+\epsilon]}$ has a canonical Whitney stratification which is a refinement of its $\mathfrak{S}$-decomposition.

Definition 2.12.([16], §I.3.3, §I.3.4) Suppose that $p$ is the only critical point of $f$ with critical value $v$.

A pair $(A, B)$ of $\mathfrak{S}$-decomposed spaces is Morse data for $f$ at $p$ if and only if there exists $\epsilon>0$ such that $v$ is the only stratified critical value of $f$ in $[v-\epsilon, v+\epsilon]$, 
and there is an $\mathfrak{S}$-decomposed embedding $i: B \rightarrow X_{\leq v-\epsilon}$ such that $X_{\leq v+\epsilon}$ is homeomorphic to $X_{\leq v-\epsilon} \cup_{B} A$ by an $\mathfrak{S}$-decomposed homeomorphism.

Coarse Morse data for $f$ at $p$ is a Whitney stratified, $\mathfrak{S}$-decomposed pair $\left(X_{[v-\epsilon, v+\epsilon]}, X_{v-\epsilon}\right)$, where $\epsilon>0$ is such that $v$ is the only stratified critical value of $f$ in $[v-\epsilon, v+\epsilon]$.

Lemma I.3.5.1 of [16] tells us that, for all sufficiently small $\delta>0, p$ is the only critical point of $f_{\left.\right|_{B_{\delta}(p) \cap X}}$ with critical value $v$ (where $B_{\delta}(p) \cap X$ is given the induced Whitney stratification), and Proposition I.3.5.3 of [16] tells us that $\mathfrak{S}$ homeomorphism type of the coarse Morse data for $f_{\left.\right|_{B_{\delta}(p) \cap X}}$ at $p$ is independent of the choice of Riemannian metric and of the choice of the sufficiently small $\delta>0$. Hence, one makes the following definition:

Definition 2.13.([16], §I.3.5) Local Morse data for $f$ at $p$ is coarse Morse data for $f_{\left.\right|_{B_{\delta}(p) \cap X}}$ at $p$ for sufficiently small $\delta>0$.

Note that local Morse data is Whitney stratified and $\mathfrak{S}$-decomposed. Also, note that the definition of local Morse data does not require that $f$ is globally proper, but only requires that the restriction of $f$ to a sufficiently small closed ball is proper.

Theorem 2.14.([16], Theorem I.3.5.4) If $p$ is the only stratified critical point of $f$ with critical value $v$, then local Morse data for $f$ at $p$ is Morse data for $f$ at $p$.

Thus, if $f$ has distinct critical values and no depraved critical points, then the change in the topology of $X$ over a given critical value of $f$ can be described in terms of local data at the corresponding nondepraved critical point. Of course, if we begin with any proper smooth function $g$ with isolated critical points, then, by Theorem 2.4, we may perturb $g$ to obtain a Morse function whose only critical points are arbitrarily close to the original critical points; in this sense, the change in the topology of $X$ over a critical value of $g$ depends only on data near the critical points. However, if the critical points of $g$ are depraved, then Lemma I.3.5.1 of [16] fails and there is no well-defined local Morse data.

We now need the notions of tangential and normal Morse data for $f$.

Definition 2.15. ([16], §I.3.6.1) Tangential Morse data for $f$ at $p$ is the Whitney stratified local Morse data for $f_{\left.\right|_{S_{p}}}$ at $p$.

Let $N$ be a normal submanifold to $X$ at $p$. Normal Morse data for $f$ at $p$ is the Whitney stratified, $\mathfrak{S}$-decomposed, local Morse data for $f_{\left.\right|_{N \cap X}}$ at $p$.

The S-decomposed homeomorphism-type of the normal Morse data is independent of the choice of the normal submanifold $N$ (see [16], I.3.6.1). 
If $N$ is a normal submanifold to $X$ at $p$ and $0<\epsilon \ll \delta \ll 1$, then there are (so far) three pairs of data at $p$ in which we are interested:

- the S-decomposed local Morse data,

$$
(A, B):=\left(B_{\delta}(p) \cap f^{-1}([v-\epsilon, v+\epsilon]), B_{\delta}(p) \cap f^{-1}(v-\epsilon)\right) ;
$$

- the tangential Morse data,

$$
(C, D):=\left(B_{\delta}(p) \cap S_{p} \cap f^{-1}([v-\epsilon, v+\epsilon]), B_{\delta}(p) \cap S_{p} \cap f^{-1}(v-\epsilon)\right) ;
$$

and

- the Whitney stratified, S-decomposed normal Morse data,

$$
(E, F):=\left(B_{\delta}(p) \cap N \cap f^{-1}([v-\epsilon, v+\epsilon]), B_{\delta}(p) \cap N \cap f^{-1}(v-\epsilon)\right) .
$$

There are two more pairs of spaces which we need to define:

- the S-decomposed, Whitney stratified upper halflink,

$$
\left(\mathfrak{l}^{+}, \partial \mathfrak{l}^{+}\right):=\left(B_{\delta}(p) \cap N \cap f^{-1}(v+\epsilon), \partial B_{\delta}(p) \cap N \cap f^{-1}(v+\epsilon)\right)
$$

and

- the S-decomposed, Whitney stratified lower halflink,

$$
\left(\mathfrak{l}^{-}, \partial \mathfrak{l}^{-}\right):=\left(B_{\delta}(p) \cap N \cap f^{-1}(v-\epsilon), \partial B_{\delta}(p) \cap N \cap f^{-1}(v-\epsilon)\right) .
$$

The homeomorphism-type of each these five pairs of spaces, with their Sdecompositions, is independent of the choice of the Riemannian metric, the normal submanifold, and the choices of $\delta$ and $\epsilon$. Note that, as each pair of spaces is intersected with a small closed ball in $M$, the definitions do not rely on the fact that we made the global assumption that $f$ is proper.

Fix a choice of $N, \delta$, and $\epsilon$ as above.

The subspace and product decompositions yield a canonical S-decomposition on the product of tangential and normal Morse data

$$
(C, D) \times(E, F)=(C \times E,(C \times F) \cup(D \times E)) .
$$

Now we can state the Main Theorem of Stratified Morse Theory.

Theorem 2.16.([16], I.3.7) There is an $\mathfrak{S}$-decomposed homeomorphism of pairs from the local Morse data of $f$ at $p$ to the product of the tangential and normal Morse data for $f$ at $p$. 
The reader should understand the importance of Theorem 2.16. Suppose for the moment that $f$ is, in fact, a (stratified) Morse function. Then, by Theorem 2.14, the change in the topology of $X$ as $f$ passes through a critical value is determined by the local Morse data at the corresponding critical point. By Theorem 2.16, this local Morse data is determined by the tangential and normal Morse data. However, the tangential Morse data is determined by the ordinary Morse theory on smooth manifolds; one finds the index of the critical point. Therefore, what Theorem 2.16 tells us is that, to understand the change in the topology of $X$, the new data that needs to be analyzed is the normal Morse data.

The following three results help one to understand the normal Morse data.

Theorem 2.17.([16], I.7.5.1) Let $\tilde{g}: M \rightarrow \mathbb{R}$ be another smooth function. Suppose that $g: X \rightarrow \mathbb{R}$ is proper, and has a nondepraved critical point at $p$.

If $d_{p} \tilde{g}$ and $d_{p} \tilde{f}$ are in the same connected component of the set $T_{S_{p}}^{*} M-$ $D_{S_{p}}^{*} M$ (i.e., the nondegenerate conormal covectors to $S_{p}$ ), then the normal Morse data and upper and lower halflinks for $g$ at $p$ are homeomorphic, by a stratumpreserving $\mathfrak{S}$-decomposed homeomorphism, to the respective data for $f$ at $p$.

Theorem 2.18.([16], I.1.8 and I.7.5.3) Let $S$ denote the connected component of $S_{p}$ which contains $p$. Suppose that $M$ is a real analytic manifold, $X$ is a subanalytic set in $M$, and $\mathfrak{S}$ is a subanalytic Whitney stratification.

Then, $T_{S}^{*} M-D_{S}^{*} M$ has a finite number of connected components, and so there are finitely many choices, up to stratum-preserving homeomorphism, for the normal Morse data, and upper and lower halflinks, of $f$ at $p$.

Theorem I.3.11.1 of [16] explains how the normal Morse data is constructed, up to $\mathfrak{S}$-homeomorphism, from the upper and lower halflinks. As a corollary, Goresky and MacPherson obtain:

Corollary 2.19.([16], I.3.11.2) The normal Morse data for $f$ at $p$ has the homotopy-type of the pair of spaces $\left(\operatorname{cone}\left(\mathfrak{l}^{-}\right), \mathfrak{l}^{-}\right)$.

\section{THE RELATIVE NONPROPER SITUATION}

In many applications, it is important to be able to eliminate the condition that the functions to which we apply Stratified Morse Theory must be proper. In other applications, it is useful to have Stratified Morse Theory relative to a map between stratified spaces. Goresky and MacPherson address these more general situations in Chapters 9 and 10 of [16], and discuss the combined generalization in Chapter 11; we present some of these results here. 
Let $Z$ be a closed Whitney stratified subset of a manifold $M$. Suppose that $f: Z \rightarrow \mathbb{R}$ is a proper smooth function, which has a nondepraved critical point $p$ with an isolated critical value $f(p)=0$.

Let $\bar{X}$ be a Whitney stratified set, with stratification $\mathfrak{S}$. Let $\bar{\pi}: \bar{X} \rightarrow Z$ be a surjective, proper, stratified map, i.e., $\bar{\pi}$ takes each stratum of $\bar{X}$ submersively to a stratum of $Z$. Let $\mathfrak{T} \subseteq \mathfrak{S}$, and let $X$ be the union of the strata in $\mathfrak{T}$.

Let $\pi:=\bar{\pi}_{\mid X}$. We are interested in the Stratified Morse Theory of $f \circ \pi: X \rightarrow \mathbb{R}$. Note that the non-relative, nonproper case is the case where $Z=\bar{X}$ and $\bar{\pi}$ is the identity map, while the relative, proper case is the case where $\mathfrak{T}=\mathfrak{S}$ (and so, $X=\bar{X})$.

Theorem 2.20.([16], I.11.2) Suppose that the interval $[a, b]$ contains no stratified critical values of the map $f$. Then, there is a $\mathfrak{T}$-decomposed homeomorphism $X_{\leq a} \cong X_{\leq b}$.

Let $(J, K)$ denote the local Morse data for $f$ at $p$. Let $(P, Q)$ denote the normal Morse data for $f$ at $p$, and let $(A, B)$ denote the tangential Morse data for $f$ at $p$.

Definition 2.21. The relative local Morse data $\left(J^{\pi}, K^{\pi}\right)$ is $\left(\pi^{-1}(J), \pi^{-1}(K)\right)$, and the relative normal Morse data $\left(P^{\pi}, Q^{\pi}\right)$ is $\left(\pi^{-1}(P), \pi^{-1}(Q)\right)$.

The following theorem says that the relative local Morse data is, in fact, Morse data for $f \circ \pi$, "at" (or, above) the point $p$.

Theorem 2.22.([16], I.11.4) For sufficiently small $\epsilon>0$, there is a T-decomposed homeomorphism

$$
X_{\leq \epsilon} \cong\left(X_{\leq-\epsilon}\right) \cup_{K^{\pi}}\left(J^{\pi}\right) .
$$

The relative nonproper version of the Main Theorem of Stratified Morse Theory is:

Theorem 2.23.([16], I.11.5) There is a T-decomposed homeomorphism

$$
\left(J^{\pi}, K^{\pi}\right) \cong(A, B) \times\left(P^{\pi}, Q^{\pi}\right) .
$$

\section{THE COMPLEX ANALYTIC SITUATION}

Throughout this subsection, we assume that $M$ is a complex analytic manifold, endowed with a Riemannian metric, $X$ is a complex analytic subset of $M$, and $\mathfrak{S}$ is a complex analytic Whitney stratification of $X$ (which always exists). We continue to assume that $f: X \rightarrow \mathbb{R}$ has a nondepraved critical point at $p \in X$. 
As in Theorem 2.18, let $S$ denote the connected component of $S_{p}$ which contains $p$. There is the following analog of Theorem 2.18.

Theorem 2.24. ([16], I.7.5.4) The space $T_{S}^{*} M-D_{S}^{*} M$ has a single connected component, and so the $\mathfrak{S}$-homeomorphism-type of the normal Morse data, and upper and lower halflinks, at the point $p$ are independent of the function $f$.

Moreover, the upper and lower halflinks are $\mathfrak{S}$-homeomorphic to each other.

In light of the theorem, in the complex analytic case, one normally speaks of the normal Morse data, and upper and lower halflinks, of $S$ at $p$, without reference to the function $f$.

We need to define one of the most basic concepts in complex analytic Stratified Morse Theory: the complex link of a stratum. Let $N \subseteq M$ be a complex analytic normal submanifold to $X$ at $p$. Note that restriction to $T_{p} N$ yields an isomorphism $\tau$ from $\left(T_{S}^{*} M\right)_{p}$ to $\left(T^{*} N\right)_{p}$.

Definition 2.25. ([16], §II.2.2) Let $\omega$ be a nondegenerate conormal covector at $p$. Let $L: N \rightarrow \mathbb{C}$ be a complex analytic map such that $L(p)=0$ and $d_{p}(\operatorname{Re} L)=$ $\tau(\omega)$.

Then, for $0<|\xi| \ll \delta \ll 1$, the complex link of $S$ in $X, \mathbb{L}_{S}$, is the $\mathfrak{S}$ decomposed (homeomorphism-type of the) space

$$
\mathbb{L}_{S}:=L^{-1}(\xi) \cap X \cap B_{\delta}(p) .
$$

The boundary of the complex link of $S$ in $X, \partial \mathbb{L}_{S}$, is the $\mathfrak{S}$-decomposed (homeomorphism-type of the) space

$$
\partial \mathbb{L}_{S}:=L^{-1}(\xi) \cap X \cap \partial B_{\delta}(p) .
$$

Using the above notation, $\mathbb{N}_{S}:=N \cap X \cap B_{\delta}(p)$ is a normal slice of $X$ at $p$, and the $\mathfrak{S}$-homeomorphism-type of $\mathbb{N}_{S}$ is determined by the $\mathfrak{S}$-homeomorphism-type of the real link of $X$ at $p$, which in turn is determined by the following result; the proof is completely constructive, but we omit the actual construction.

Theorem 2.26.([16], §II.2.5) The S-homeomorphism-type of the real link of $S$ at $p$ and normal Morse data of $S$ at $p$ are determined by a monodromy (Sdecomposed) homeomorphism of $\mathbb{L}_{S}$, obtained by letting $\xi$ travel around a small circle around the origin, and leaving a neighborhood of $\partial \mathbb{L}_{S}$ fixed.

Note that if $S$ is a maximal stratum of $X$ (i.e., not contained in the closure of another stratum), then $\mathbb{N}_{S}=\{p\}$ and $\mathbb{L}_{S}=\emptyset$. Even in this trivial case, 
Theorem 2.26 remains true, for the real link is empty, and the normal Morse data is $(\{p\}, \emptyset)$.

We have the following strong uniqueness theorem.

Theorem 2.27.([16], II.2.3) The S-homeomorphism-types of $\mathbb{L}_{S}, \partial \mathbb{L}_{S}$, and $\mathbb{N}_{S}$ are independent of the point $p \in S$, the nondegenerate conormal covector $\omega$, the Riemannian metric, the normal submanifold $N$, the function $L$, and the choice of $\delta$ and $\xi$ (provided $0<|\xi| \ll \delta \ll 1$ ).

Finally, the complex link determines the normal Morse data up to homotopy.

Theorem 2.28.([16], II.2.4) If the critical point $p$ of $f$ is nondegenerate, then the normal Morse data of $f$ at $p$ has the homotopy-type of the pair $\left(\mathbb{N}_{S}, \mathbb{L}_{S}\right)$, which is also the homotopy-type of the pair $\left(\operatorname{cone}\left(\mathbb{L}_{S}\right), \mathbb{L}_{S}\right)$.

\section{CONSTRUCTIBLE COMPLEXES OF SHEAVES}

In [16], 6.A, Goresky and MacPherson give a very brief discussion of the connection between Stratified Morse Theory and hypercohomology of bounded, constructible complexes of sheaves. Background references for this subsection are [1], [20], [6], [39], and [31], Appendix B.

Let $X$ be a complex analytic space, embedded in a complex manifold $M$, and let $\mathfrak{S}:=\left\{S_{\alpha}\right\}_{\alpha}$ be a complex analytic Whitney stratification of $X$. For notational convenience, we assume that each stratum of $\mathfrak{S}$ is connected. Fix a Riemannian metric on $M$.

Fix a base ring $R$ that is a regular Noetherian ring with finite Krull dimension (e.g., $\mathbb{Z}, \mathbb{Q}$, or $\mathbb{C}$ ). Let $\mathbf{F}^{\bullet}$ be a bounded complex of sheaves of $R$-modules, which is constructible with respect to $\mathfrak{S}$.

Let $p \in X$, let $S$ denote the stratum containing $p$, and let $d_{S}:=\operatorname{dim}_{\mathbb{C}} S$. As in Definition 2.25, let $\left(\mathbb{N}_{S}, \mathbb{L}_{S}\right)$ be the complex normal slice and complex link of $S$ in $X$.

One then has the following hypercohomology version of the Main Theorem of Stratified Morse Theory.

Theorem 2.29.([16], II.6.A.1) For all $k$, the degree $k$ Morse module given by the hypercohomology

$$
m_{S}^{k}\left(\mathbf{F}^{\bullet}\right):=\mathbb{H}^{k}\left(\mathbb{N}_{S}, \mathbb{L}_{S} ; \mathbf{F}_{\mathbb{N}_{S}}^{\bullet}\left[-d_{S}\right]\right)=\mathbb{H}^{k-d_{S}}\left(\mathbb{N}_{S}, \mathbb{L}_{S} ; \mathbf{F}^{\bullet}\right),
$$

is independent, up to isomorphism, of the choices of $\mathbb{N}_{S}, \mathbb{L}_{S}$, and $p \in S$. 
Suppose that $\tilde{f}: M \rightarrow \mathbb{R}$ is a smooth function such that $f:=\tilde{f}_{\left.\right|_{X}}$ is a Morse function, and such that the interval $[a, b] \subseteq \mathbb{R}$ contains no stratified critical values of $f$ other than $v:=f(p)$. Then, for all $k$, there is an isomorphism

$$
\mathbb{H}^{k}\left(X_{\leq b}, X_{\leq a} ; \mathbf{F}^{\bullet}\right) \cong \mathbb{H}^{k-\lambda}\left(\mathbb{N}_{S}, \mathbb{L}_{S} ; \mathbf{F}^{\bullet}\right)=m_{S}^{k-\lambda+d_{S}}\left(\mathbf{F}^{\bullet}\right),
$$

where $\lambda$ is the index of $f_{\left.\right|_{S}}$ at $p$.

Note that our notation and indexing differs slightly from that in [16]; we are using our conventions from [30] and [32].

Remark 2.30. Suppose that $\mathbf{P}^{\bullet}$ is a perverse sheaf (which is actually a complex of sheaves) on $X$, constructible with respect to $\mathfrak{S}$. This is equivalent to $\mathbf{P}^{\bullet}$ being pure (with shift 0). See [19] and [16], II.6.A.3. Because we have included the shift by $-d_{S}$ in the definition of the Morse modules, purity implies that perverse sheaves have possibly non-zero Morse modules in only degree zero. In particular, this applies to the (middle perversity) intersection cohomology complex with constant coefficients, or with coefficients in a local system.

Theorem 2.29 is a theorem about a real function $f$. There is a corresponding complex analytic result. Suppose now that $f:(X, p) \rightarrow(\mathbb{C}, 0)$ is a complex analytic function. By definition, the stalk cohomology at $p$ of the vanishing cycles of $\mathbf{F}^{\bullet}$ along $f, \phi_{f} \mathbf{F}^{\bullet}$, is given by

$$
H^{k}\left(\phi_{f} \mathbf{F}^{\bullet}\right)_{p} \cong \mathbb{H}^{k+1}\left(B_{\delta}(p) \cap X, B_{\delta}(p) \cap f^{-1}(\xi) ; \mathbf{F}^{\bullet}\right),
$$

where $0<|\xi| \ll \delta \ll 1$, and one may replace the closed ball $B_{\delta}$ with the open ball. The space $B_{\delta}(p) \cap f^{-1}(\xi)$ is the Milnor fiber of $f$ at $p, F_{f, p}$. If $\mathbf{F}^{\bullet}$ is the constant sheaf $\mathbb{Z}_{X}^{\bullet}$, then, since $B_{\delta}(p) \cap X$ is contractible, the isomorphism above becomes the well-known formula

$$
H^{k}\left(\phi_{f} \mathbb{Z}_{X}^{\bullet}\right)_{p} \cong H^{k+1}\left(B_{\delta}(p) \cap X, F_{f, p} ; \mathbb{Z}\right) \cong \widetilde{H}^{k}\left(F_{f, p} ; \mathbb{Z}\right),
$$

where $\widetilde{H}^{k}$ denotes reduced reduced cohomology and, by convention, $\widetilde{H}^{0}(\emptyset ; \mathbb{Z}) \cong$ $\mathbb{Z}$. Because of the shift of degrees in $(\dagger)$, it is usually nicer to consider the shifted vanishing cycles $\phi_{f} \mathbf{F}^{\bullet}[-1]$, so that $H^{k}\left(\phi_{f} \mathbf{F}^{\bullet}[-1]\right)_{p}=H^{k-1}\left(\phi_{f} \mathbf{F}^{\bullet}\right)_{p}$. See [6], p. 105-106, and [31], Appendix B, p.227-228.

By applying Theorem 2.29 to $|f-\xi|^{2}$, one obtains:

Theorem 2.31.([16], II6.A.2) Let $\tilde{f}:(M, p) \rightarrow(\mathbb{C}, 0)$ be a complex analytic function such that $d_{p} \tilde{f}$ is a nondegenerate conormal covector to $S$ at $p$, and such that the complex Hessian of $\tilde{f}_{\left.\right|_{S}}$ at $p$ is nondegenerate. Let $f:=\tilde{f}_{\left.\right|_{X}}$.

Then, for all $k, H^{k}\left(\phi_{f} \mathbf{F}^{\bullet}[-1]\right)_{p} \cong m_{S}^{k}\left(\mathbf{F}^{\bullet}\right)$, i.e., for $0<|\xi| \ll \delta \ll 1$,

$$
\mathbb{H}^{k}\left(B_{\delta}(p) \cap X, B_{\delta}(p) \cap f^{-1}(\xi) ; \mathbf{F}^{\bullet}\right) \cong \mathbb{H}^{k-d_{S}}\left(\mathbb{N}_{S}, \mathbb{L}_{S} ; \mathbf{F}^{\bullet}\right) \text {. }
$$




\section{3. "Classical" Applications}

In this section, we will recall some of the important results that Goresky and MacPherson obtained using Stratified Morse Theory. We present these results in the chronological order in which they appeared: first, in Morse Theory and Intersection Homology Theory [14], then in the paper Stratified Morse Theory [15] and, finally, in the book [16], which, in addition to containing results not found in the two papers, also contains detailed proofs of the results from the earlier two works.

\section{INTERSECTION HOMOLOGY}

Intersection homology and cohomology were developed by Goresky and MacPherson. Their first paper on the subject was [12], and their sheaf-theoretic presentation and results appeared in [13]. Intersection cohomology is important not just because it provides useful topological data about a space, but also because it arises in the Weil conjectures for singular varieties, in the Decomposition Theorem of Beilinson, Bernstein, Deligne, and Gabber (see [1]), and the proof of the Kazhdan-Lusztig conjecture in representation theory. For nice expositions of these applications, the reader is directed to [24], and [23].

Let $I H_{k}$ denote the degree $k$ intersection homology group (with middle perversity) with integral coefficients. (Here, we use the topological indexing, as in [12], on intersection homology; hence, the non-zero homology occurs in non-negative degrees.)

Let $X$ be a complex analytic space with a complex analytic Whitney stratification. Let $p \in X$, and let $S$ denote the connected component of the stratum containing $p$. Suppose that $f: X \rightarrow \mathbb{R}$ is a Morse function with a critical point at $p$. Let $v:=f(p)$.

By using properties of intersection homology, and using the same description of the normal Morse data that is used in proving Theorem 2.26, Goresky and MacPherson obtained:

Theorem 3.1.([14], 4.1; [16], II.6.4) Let $i=\lambda+c$, where $c$ is the complex codimension of $S$ in $X$, and $\lambda$ is the Morse index of $f_{\left.\right|_{S}}$ at $p$.

Then, for all sufficiently small $\epsilon>0$, for all $k \neq i, I H_{k}\left(X_{\leq v+\epsilon}, X_{\leq v-\epsilon}\right)=0$. In addition, $A_{p}:=I H_{i}\left(X_{\leq v+\epsilon}, X_{\leq v-\epsilon}\right)$ is free Abelian and, up to isomorphism, is independent of the point $p$ in $S$ and independent of $f$ itself.

Remark 3.2. The group $A_{p}$ is called the Morse group of $S$ in $X$. This is the homological version of the Morse module (over $\mathbb{Z}$ ) from Theorem 2.29, when one 
uses the intersection cohomology complex for $\mathbf{F}^{\bullet}$. As this intersection cohomology complex is perverse, the Morse module is possibly non-zero only in degree 0 ; see Remark 2.30.

Goresky and MacPherson show, in fact, that, if $c>0$, then the Morse group is isomorphic to the image of the variation map

$$
\text { var : } I H_{c-1}\left(\mathbb{L}_{S}, \partial \mathbb{L}_{S}\right) \rightarrow I H_{c-1}\left(\mathbb{L}_{S}\right),
$$

determined by the monodromy of the complex link of $S$. Note that it is possible that $A_{p}=0$.

We should remark that Theorem 6.4 of [16] is actually more general than the result appearing in [14]. In [16], Goresky and MacPherson allow for the case where $X$ is an open dense union of strata of a larger stratified space $Z$, and $p \in Z-X$ is a critical point of a proper Morse function $f: Z \rightarrow \mathbb{R}$.

As a quick corollary to Theorem 3.1, Goresky and MacPherson obtained the following Lefschetz hyperplane theorem for intersection homology, which was their original motivation for developing Stratified Morse Theory.

Theorem 3.3.([14], 5.4; [16], II.6.10) Let $X$ be a complex n-dimensional algebraic subvariety of some complex projective space $\mathbb{P}^{N}$. Let $H$ be a hyperplane in $\mathbb{P}^{N}$ which is transverse to each stratum of some complex analytic Whitney stratification of $X$.

Then, the inclusion $X \cap H \hookrightarrow X$ induces isomorphisms $I H_{k}(X \cap H) \cong I H_{k}(X)$, for all $k<n-1$, and a surjection $I H_{n-1}(X \cap H) \rightarrow I H_{n-1}(X)$.

Proof. Fix a complex analytic Whitney stratification of $X$ such that $H$ is transverse to all of the strata. Let $f: X \rightarrow \mathbb{R}$ be a Morse perturbation of the function which gives the square of the distance to $H$, with respect to the Fubini-Study metric on $\mathbb{P}^{N}$. Then, by the transversality assumption on $H$, for some small $\delta>0, X_{<\delta}$ is homeomorphic to $Y \times \mathbb{R}^{2}$. In addition, $X_{<m}=X$ for some $m>0$.

By the Künneth formula and the long exact sequence of a pair for intersection homology, what we need to show is that $I H_{k}\left(X_{<m}, X_{<\delta}\right)=0$ for all $k<n$.

However, this follows at once from Theorem 3.1, since the index $i$ of $f_{\left.\right|_{S}}$ at a critical point $p$ in a stratum $S$ must be at least $\operatorname{dim}_{\mathbb{C}} S$.

Remark 3.4. As one sees in the proof, one can eliminate the assumption that $H$ is transverse to all of the strata if one is willing to replace $X \cap H$ by a neighborhood of $X \cap H$. This generalization is what appears in [16], II.6.10, where the result is further generalized to apply to the relative case of a purely $n$-dimensional algebraic variety and a (not necessarily proper) algebraic map $\pi: X \rightarrow \mathbb{P}^{N}$ with finite fibers. 
In [34], Theorem 7.2, Milnor applies Morse Theory to prove that a Stein manifold of complex dimension $k$ has the homotopy-type of a CW complex of dimension $k$, and so, its homology vanishes above dimension $k$. Goresky and MacPherson used Stratified Morse Theory to obtain an analogous result about intersection homology.

Theorem 3.5.([14], 5.3; [16], II.6.9) Let $X$ be an $n$-dimensional Stein space. Then, for all $k>n, I H_{k}(X)=0$, and $I H_{n}(X)$ is torsion-free.

Proof. Embed $X$ as a closed analytic subspace of some $\mathbb{C}^{N}$. Fix a complex analytic Whitney stratification of $X$. Choose a generic point $q \in \mathbb{C}^{N}$, and let $f$ be the Morse function on $X$ given by the square of the Euclidean distance to $q$.

Then, if $p$ is in a stratum $S$ and $p$ is a critical point of $f_{\mid S}$, then the index of $f_{\mid S}$ at $p$ is at most $\operatorname{dim}_{\mathbb{C}} S$. Thus, by Theorem 3.1, if $v=f(p)$ and $\epsilon>0$ is sufficiently small, for all $k>n, I H_{k}\left(X_{\leq v+\epsilon}, X_{\leq v-\epsilon}\right)=0$, and, for all $k \leq n$, $I H_{k}\left(X_{\leq v+\epsilon}, X_{\leq v-\epsilon}\right)$ is torsion-free. The result follows now by induction and the long exact sequence of a pair in intersection homology.

Let $g: X \rightarrow \mathbb{C}$ be a complex analytic map. In the category of perverse sheaves on $X$ (see [1], [20]), the simple objects are intersection cohomology sheaves with coefficients in irreducible local systems. By applying Stratified Morse Theory to this intersection cohomology, and using a careful topological description of how the nearby fiber collapses to $g^{-1}(0)$, Goresky and MacPherson gave the first proof of the following result.

Theorem 3.6. ([14], 6.6) If $\mathbf{P}^{\bullet}$ is a perverse sheaf on $X$, then the shifted nearby cycles $\psi_{g} \mathbf{P}^{\bullet}[-1]$ are a perverse sheaf on $g^{-1}(0)$.

Remark 3.7. Goresky and MacPherson make the assumption that $g: X \rightarrow \mathbb{C}$ is proper. This assumption is not necessary; see, for instance, [20], X.10.3.13. In our statement of the theorem, we have used the now standard indexing on perverse sheaves which places possibly non-zero cohomology in only non-positive degrees. This is why we must include the shift by -1 in our statement. Finally, Goresky and MacPherson used the term "vanishing cycles" for $\psi_{g}$. In modern terminology, the $\psi_{g}$ functor that appears in [14] is called the "nearby cycles" functor.

\section{HOMOTOPY RESULTS}

In [15], Chapter 4, and in [16], II.1, Goresky and MacPherson use relative Stratified Morse Theory to obtain a number of important results on homotopy types and homotopy groups. Here, we present three such results, so that the reader can get the flavor of the theorems. 
By convention, below, we set the dimension of the empty set to be $-\infty$.

Theorem 3.8.([15], 4.1; [16], II.1.1) Let $X$ be an $n$-dimensional nonsingular, connected, complex algebraic variety. Let $\pi: X \rightarrow \mathbb{P}^{N}$ be an algebraic map and let $H \subseteq \mathbb{P}^{N}$ be a linear subspace of complex codimension c. Let $H_{\delta}$ be the $\delta$ neighborhood of $H$ with respect to some real analytic Riemannian metric. Let $\phi(k)=\operatorname{dim}\left\{y \in \mathbb{P}^{n}-H \mid \operatorname{dim} \pi^{-1}(y)=k\right\}$.

Then, if $\delta>0$ is sufficiently small, the homomorphism $\pi_{i}\left(\pi^{-1}\left(H_{\delta}\right)\right) \rightarrow \pi_{i}(X)$ is an isomorphism for all $i<\hat{n}$, and is a surjection for $i=\hat{n}$, where

$$
\hat{n}:=n-1-\sup _{k}[2 k-(n-\phi(k))+\inf (\phi(k), c-1)] .
$$

Furthermore, if $H$ is generic or $\pi$ is proper, then $\pi^{-1}\left(H_{\delta}\right)$ may be replaced by $\pi^{-1}(H)$.

Theorem 3.9.([15], 4.1*; [16], II.1.1*) Let $X$ be an $n$-dimensional (possibly singular) complex analytic variety. Let $\pi: X \rightarrow \mathbb{P}^{N}-H$ be a proper complex analytic map, where $H \subseteq \mathbb{P}^{N}$ is a linear subspace of codimension $c$. Let $\phi(k)=\operatorname{dim}\left\{y \in \pi(X) \mid \operatorname{dim} \pi^{-1}(y)=k\right\}$.

Then, $X$ has the homotopy-type of a $C W$ complex of real dimension less than or equal to

$$
\hat{n}^{*}:=n+\sup _{k}[2 k-(n-\phi(k))+\inf (\phi(k), c-1)] .
$$

Theorem 3.10.([16], II.1.2) Let $X$ be a complex algebraic subvariety of some complex algebraic manifold $M$. Let $\pi: X \rightarrow \mathbb{P}^{N}$ be a (not necessarily proper) complex algebraic map with finite fibers. Let $H \subseteq \mathbb{P}^{N}$ be a linear subspace of complex codimension $c$. Let $H_{\delta}$ be the $\delta$-neighborhood of $H$ with respect to some real analytic Riemannian metric. Let $\phi(k)$ denote the dimension of the set of points $p \in X-\pi^{-1}(H)$ such that a neighborhood of $p$ in $X$ can be defined in $M$ by $k$ equations and no fewer.

Then, if $\delta>0$ is sufficiently small, the homomorphism $\pi_{i}\left(\pi^{-1}\left(H_{\delta}\right)\right) \rightarrow \pi_{i}(X)$ is an isomorphism for all $i<\hat{n}$, and is a surjection for $i=\hat{n}$, where

$$
\hat{n}:=-1+\inf _{k}\left[\operatorname{dim}_{\mathbb{C}} M-k+\inf (\phi(k), c-1)\right] .
$$

\section{COMPLEMENTS OF AFFINE SUBSPACES}

In Part III of [16], Goresky and MacPherson study the topology of the complement of an affine subspace arrangement, i.e.,

$$
M:=\mathbb{R}^{n}-\bigcup_{i=1}^{m} A_{i}
$$


where $\mathcal{A}:=\left\{A_{1}, \ldots, A_{m}\right\}$ is a finite collection of affine linear subspaces of $\mathbb{R}^{n}$. They arrive at a combinatorial description of the homology groups of $M$. This generalized work of Zaslavsky in [43] and Orlik and Solomon in [35]. We will briefly describe Goresky and MacPherson's result.

Associated to $\mathcal{A}$, there is a partially ordered set $\mathcal{P}$, whose elements $v$ consist of the flats of $\mathcal{A}$, i.e., non-empty, finite intersections $A_{i_{1}} \cap \cdots \cap A_{i_{r}}$. The partial order on this set is given by containment: if $v, w \in \mathcal{P}$, then $v \leq w$ if and only if $v \subseteq w$. There is a maximum element, corresponding to the intersection of no subspaces, $T:=\mathbb{R}^{n} \in \mathcal{P}$. For $v \in \mathcal{P}$, define the ranking function $d(v):=\operatorname{dim}_{\mathbb{R}}(v)$.

We should remark that it is only the structure of $\mathcal{P}$ as an ordered set, together with its ranking function $d$, that will be used here. To emphasize this fact, Goresky and MacPherson write $|v|$ where we are writing simply $v$, and use $v$ to denote an abstract element of the partially ordered set.

It turns out that the homology of $M$ can be described solely in terms of the partially ordered set $\mathcal{P}$, and the ranking function $d$, by using order complexes.

Let $\mathcal{S}$ be an arbitrary ordered set. The order complex $K(\mathcal{S})$ is a simplicial complex with one vertex for every element $v \in \mathcal{S}$ and one $k$ simplex for every chain $v_{0}<v_{1}<\cdots<v_{k}$ of elements of $\mathcal{S}$. For $v, w \in \mathcal{S}$, let $\mathcal{S}_{>v}:=\{x \in \mathcal{S} \mid x>v\}$, and let $\mathcal{S}_{(v, w)}:=\{x \in \mathcal{S} \mid v<x<w\}$.

To apply Stratified Morse Theory to this situation, Goresky and MacPherson consider all of $\mathbb{R}^{n}$ as a Whitney stratified space, where the Whitney stratification is determined by the flats. For each $v \in \mathcal{P}$, define the stratum $S(v)$ by

$$
S(v):=v-\bigcup_{w<v} w .
$$

It is trivial to verify that this yields a Whitney stratification of $\mathbb{R}^{n}$.

Now, one uses Theorem 2.5, and selects "almost any" $q \in \mathbb{R}^{n}$ such that the distance-squared function $r_{q}: \mathbb{R}^{n} \rightarrow \mathbb{R}$ is a stratified Morse function, with respect to the Whitney stratification above. By analyzing the Stratified Morse Theory of the nonproper function $\left(r_{q}\right)_{\left.\right|_{M}}$, Goresky and MacPherson prove:

Theorem 3.11.([16], III.1.3) The homology of $M$ is given by

$$
H_{i}(M ; \mathbb{Z}) \cong \bigoplus_{v \in \mathcal{P}} H^{n-d(v)-i-1}\left(K\left(\mathcal{P}_{>v}\right), K\left(\mathcal{P}_{(v, T)}\right) ; \mathbb{Z}\right),
$$

where, by convention, $H^{-1}(\emptyset, \emptyset ; \mathbb{Z})=\mathbb{Z}$, i.e., $T$ contributes a copy of $\mathbb{Z}$ to the homology group $H_{0}(M ; \mathbb{Z})$. 
As the complexified arrangement $\mathcal{A} \otimes \mathbb{C}$ has the "same" (isomorphic) partially ordered set $\mathcal{P}$, but a different ranking function, one concludes:

Corollary 3.12.([16], III.1.4) Letting $b_{k}$ denote the $k$-th Betti number,

$$
\sum_{k=0}^{n} b_{k}\left(\mathbb{R}^{n}-\bigcup_{i=1}^{m} A_{i}\right)=\sum_{k=0}^{2 n} b_{k}\left(\mathbb{C}^{n}-\bigcup_{i=1}^{m} A_{i} \otimes \mathbb{C}\right) .
$$

Since the appearance of [16], there have been a number of substantial improvements to Theorem 3.11; see [2], [3], [7], and [44].

\section{4. "Modern" Developments}

In this section, we will discuss a sampling of results related to Stratified Morse Theory which have appeared since the publication of [16].

\section{COMPLEX HYPERPLANE ARRANGEMENTS}

We wish to describe some of the results that D. Cohen obtained in his 1992 doctoral dissertation [4]. An extensive treatment of the relations between Stratified Morse Theory and hyperplane arrangements can be found in Stratified Morse Theory in Arrangements, [5], in these volumes.

Definition 4.1. Suppose that $X$ is a Whitney stratified subset of a smooth manifold $M$. A stratified Morse function $f: X \rightarrow \mathbb{R}$ is weakly self-indexing (with respect to the stratification) if and only if, for all strata $S_{\alpha}$ and $S_{\beta}$, if $\operatorname{dim} S_{\alpha}>\operatorname{dim} S_{\beta}$, and $v_{\alpha}$ and $v_{\beta}$ are critical values of $f_{\left.\right|_{S_{\alpha}}}$ and $f_{\left.\right|_{\beta}}$, respectively, then $v_{\alpha}<v_{\beta}$.

In other words, $f$ is weakly self-indexing if and only if, as the value of $f$ grows, one hits critical points on higher-dimensional strata before hitting critical points on lower-dimensional strata.

As in the last subsection of Section 3 , let $\mathcal{A}:=\left\{A_{1}, \ldots, A_{m}\right\}$ be an affine subspace arrangement in $X:=\mathbb{R}^{n}$, and stratify $\mathbb{R}^{n}$ by the Whitney stratification determined by the flats. Let $p:=\left(p_{1}, \ldots, p_{n}\right) \in \mathbb{R}^{n}-\bigcup_{k=1}^{m} A_{k}$.

Cohen proves:

Proposition 4.2.([4], 1.2) There exist positive constants $\omega_{1}, \ldots, \omega_{n}$ such that the function $f: \mathbb{R}^{n} \rightarrow \mathbb{R}$ given by $f\left(x_{1}, \ldots, x_{n}\right)=\sum_{k=1}^{d} \omega_{k}\left(x_{k}-p_{k}\right)^{2}$ is weakly self-indexing, and has a unique critical point of index zero (a minimum) on each stratum. 
Fix such a set of $\omega_{k}$ and $f$.

As $f$ is weakly self-indexing, one obtains a filtration

$$
\emptyset=X_{\leq-1} \subseteq X_{\leq w_{n}} \subseteq X_{\leq w_{n-1}} \subseteq \cdots \subseteq X_{\leq w_{0}}=X=\mathbb{R}^{n},
$$

where each $w_{k}$ is greater than all of the critical values of $f$ on all $k$-dimensional strata, but less than all the critical values on $(k-1)$-dimensional strata.

Suppose now that we consider the case of a complex hyperplane arrangement: $X:=\mathbb{C}^{n}$ and $\mathcal{A}$ is an arrangement of complex hyperplanes in $X$. Once again use the flats of $\mathcal{A}$ to produce a (complex analytic) Whitney stratification $\mathfrak{S}$ of $X$. Let $M:=X-\bigcup_{k=1}^{m} A_{k}$, and let $i: M \hookrightarrow X$ denote the inclusion.

Let $\mathbf{P}^{\bullet}$ be a perverse sheaf on $X$ (over $\mathbb{Z}$ or $\mathbb{C}$, or more general base rings), which is constructible with respect to the stratification $\mathfrak{S}$. Interesting examples to use for $\mathbf{P}^{\bullet}$ include $R i_{*} \mathbb{Z}_{M}^{\bullet}[n]$ and the intersection cohomology complex, $I C_{X}^{\bullet}(\mathcal{L})$, with coefficients in a local system $\mathcal{L}$ on $M$.

One may consider the underlying real arrangement, and apply Proposition 4.2 and $(\dagger)$ to produce a weakly self-indexing Morse function $f: X \rightarrow \mathbb{R}$, together with a filtration

$$
\emptyset=X_{-1} \subseteq X_{\leq w_{n}} \subseteq X_{\leq w_{n-1}} \subseteq \cdots \subseteq X_{\leq w_{0}}=X=\mathbb{C}^{n},
$$

where each $w_{k}$ is greater than all of the critical values of $f$ on all strata of complex dimension $k$, but less than all the critical values on strata of complex dimension $(k-1)$.

As the index of each critical point of $f$ is 0 , when we apply Theorem 2.29, we conclude that, if $k \neq-l$, then $\mathbb{H}^{k}\left(X_{\leq w_{l}}, X_{\leq w_{l+1}} ; \mathbf{P}^{\bullet}\right)=0$, and

$$
C^{-l}\left(\mathbf{P}^{\bullet}\right):=\mathbb{H}^{-l}\left(X_{\leq w_{l}}, X_{\leq w_{l+1}} ; \mathbf{P}^{\bullet}\right) \cong \bigoplus_{\operatorname{dim} S=l} m_{S}^{0}\left(\mathbf{P}^{\bullet}\right) .
$$

Now, the connecting homomorphism in the long exact sequence on hypercohomology of the triple $\left(X_{\leq w_{l-1}}, X_{\leq w_{l}}, X_{\leq w_{l+1}}\right)$ yields a map $\partial^{-l}: C^{-l}\left(\mathbf{P}^{\bullet}\right) \rightarrow$ $C^{-l+1}\left(\mathbf{P}^{\bullet}\right)$ such that $\partial^{-l+1} \circ \partial^{-l}=0$, i.e., $\partial^{*}$ is a differential.

The main theorem of $[4]$ is:

Theorem 4.3.([4], 2.4) The $k$-th hypercohomology module $\mathbb{H}^{k}\left(X ; \mathbf{P}^{\bullet}\right)$ is isomorphic to the degree $k$ cohomology of the differential complex

$$
0 \rightarrow C^{-n}\left(\mathbf{P}^{\bullet}\right) \stackrel{\partial^{-n}}{\longrightarrow} C^{-n+1}\left(\mathbf{P}^{\bullet}\right) \stackrel{\partial^{-n+1}}{\longrightarrow} \cdots \stackrel{\partial^{-1}}{\longrightarrow} C^{0}\left(\mathbf{P}^{\bullet}\right) \rightarrow 0,
$$

in which $C^{-l}\left(\mathbf{P}^{\bullet}\right) \cong \bigoplus_{\operatorname{dim} S=l} m_{S}^{0}\left(\mathbf{P}^{\bullet}\right)$. 
When $\mathbf{P}^{\bullet}=I C_{X}^{\bullet}(\mathcal{L})$, this theorem provides information about the intersection cohomology $I H^{k}(X ; \mathcal{L})$.

When $\mathbf{P}^{\bullet}=R i_{*} \mathbb{Z}_{M}^{\bullet}[n], \mathbb{H}^{k}\left(X ; \mathbf{P}^{\bullet}\right) \cong H^{k+n}(M ; \mathbb{Z})$, and the Morse modules $m_{S}^{0}\left(\mathbf{P}^{\bullet}\right)$ are isomorphic to $\mathbb{Z}^{|\mu(S)|}$, where $\mu(S)$ is the Möbius function of the flat $\bar{S}$ (see [4] or [36]). Using the techniques of Theorem 3.11, Cohen shows that, in this case, the differentials in the complex in Theorem 4.3 are zero and, hence, he recovers the result of Orlik and Solomon [35] that the cohomology $H^{*}(M ; \mathbb{Z})$ is free Abelian in each degree, with ranks given by sums of absolute values of the Möbius functions of the flats of the appropriate dimension.

The global topology of complex hyperplane arrangements can be reduced to a local study, by taking the complex "cone" on the arrangement - that is, by homogenizing the defining equation of the arrangement. Then, one may study the topology of the original arrangement by analyzing, near the origin, the topology of the new, central arrangement (one in which each hyperplane contains the origin), and the complex appearing in Theorem 4.3 can be generalized, locally, for a perverse sheaf on any complex analytic space, with any complex analytic Whitney stratification. This generalization appears in [28]. We will briefly describe this result.

Since we wish to work locally, we may assume that we have a $d$-dimensional complex analytic $X$, embedded in an open subset $\mathcal{U} \subseteq \mathbb{C}^{n+1}$, and that our point of interest is the origin $\mathbf{0} \in X$. Let $\mathbf{P}^{\bullet}$ be a perverse sheaf of $R$-modules on $X$, constructible with respect to some complex analytic Whitney stratification $\mathfrak{S}$ (with connected strata).

For a generic (with respect to $\mathfrak{S}$ ) choice of linear forms $z_{0}, \ldots, z_{n}$ on $\mathbb{C}^{n+1}$, we consider iterated nearby and vanishing cycles along these functions, composed with a shift by -1 . Thus, we consider $\psi_{z_{k}}[-1]$ and $\phi_{z_{k}}[-1]$, where we do not distinguish in the notation between $z_{k}$ and its restriction to various subspaces. (In fact, it is only the corresponding differentials $d_{\mathbf{0}} z_{0}, \ldots d_{\mathbf{0}} z_{n}$ which matter.)

For all $k$, let

$$
\gamma^{k}\left(\mathbf{P}^{\bullet}\right):=\phi_{z_{k}}[-1] \psi_{z_{k-1}}[-1] \ldots \psi_{z_{0}}[-1] \mathbf{P}^{\bullet},
$$

where we mean that $\gamma^{0}\left(\mathbf{P}^{\bullet}\right):=\phi_{z_{0}}[-1] \mathbf{P}^{\bullet}$. Each $\gamma^{k}\left(\mathbf{P}^{\bullet}\right)$ is a perverse sheaf which, by the genericity of the $z_{i}$ 's, has the origin as an isolated point in its support. Thus, the stalk cohomology of $\gamma^{k}\left(\mathbf{P}^{\bullet}\right)$ at the origin is zero outside of degree 0 . Let $A^{k}\left(\mathbf{P}^{\bullet}\right):=H^{0}\left(\gamma^{k}\left(\mathbf{P}^{\bullet}\right)\right)_{\mathbf{0}}$. Essentially by looking at long exact sequences of triples, as in the construction of the differentials in Theorem 4.3, one obtains a canonical map $\partial^{k}: A^{k}\left(\mathbf{P}^{\bullet}\right) \rightarrow A^{k+1}\left(\mathbf{P}^{\bullet}\right)$ such that $\partial^{k+1} \circ \partial^{k}=0$, and it is easy to show: 
Theorem 4.4. ([28], Theorem 5.4) The degree $k$ stalk cohomology $H^{k}\left(\mathbf{P}^{\bullet}\right)_{\mathbf{0}}$ is isomorphic to the degree $k$ cohomology of the differential complex

$$
0 \rightarrow A^{-d}\left(\mathbf{P}^{\bullet}\right) \stackrel{\partial^{-d}}{\longrightarrow} A^{-d+1}\left(\mathbf{P}^{\bullet}\right) \stackrel{\partial^{-d+1}}{\longrightarrow} \cdots \stackrel{\partial^{-1}}{\longrightarrow} A^{0}\left(\mathbf{P}^{\bullet}\right) \rightarrow 0 .
$$

In addition, one can describe the modules $A^{k}\left(\mathbf{P}^{\bullet}\right)$ in terms of the Morse modules of strata and the relative polar varieties of Lê and Teissier [27].

Theorem 4.5.([28], 7.5; [32], 4.24) For each $k$, the module $A^{-k}\left(\mathbf{P}^{\bullet}\right)$ is isomorphic to

$$
\bigoplus_{S \in \mathfrak{S}}\left(m_{S}^{0}\left(\mathbf{P}^{\bullet}\right) \otimes R^{\gamma_{S}^{k}}\right)
$$

where $R$ is the base ring, and $\gamma_{S}^{k}$ is the multiplicity of the $k$-dimensional polar variety of $\bar{S}$ at the origin.

Remark 4.6. Note that, in the case of a central arrangement, if $S \in \mathfrak{S}$, the only non-zero $\gamma_{S}^{k}$ occurs when $k=\operatorname{dim} S$, and $\gamma_{S}^{\operatorname{dim} S}=1$. Thus, the characterization of the modules in Theorem 4.5 generalizes that of Theorem 4.3.

We should also remark that the summands in Theorem 4.5 are products of a normal factor $m_{S}^{0}\left(\mathbf{P}^{\bullet}\right)$ and a tangential factor $R^{\gamma_{S}^{k}}$. We shall discuss further generalizations of Theorem 2.29, the Main Theorem of Stratified Morse Theory for hypercohomology, later in this section.

\section{SELF-INDEXING STRATIFIED MORSE FUNCTIONS}

In this subsection, we will discuss the work of M. Grinberg ([17], 2005) on self-indexing stratified Morse functions. As the names imply, this is a stronger form of the notion of weakly self-indexing from the previous subsection.

Throughout this subsection, we weaken our requirements on a (stratified) Morse function; we do not require that distinct critical points have distinct critical values.

Suppose that $M$ is an $n$-dimensional compact, smooth manifold, and that $f: M \rightarrow \mathbb{R}$ is a Morse function. We write $\Sigma f$ for the critical locus of $f$.

Definition 4.7. The Morse function $f$ is self-indexing if and only if, for all $p \in \Sigma f$, the index of $f$ at $p$ is equal to $f(p)$.

If $f$ is a self-indexing Morse function, one may perturb $f$ slightly to obtain a Morse function with distinct critical values, but for which all of the critical points of index 0 have values arbitrarily close to 0 , all of the critical points of index 1 have value arbitrarily close to 1 , and so on. 
The importance of $f$ being a self-indexing Morse function is that it yields a filtration

$$
\emptyset=M_{-0.5} \subseteq M_{\leq 0.5} \subseteq M_{\leq 1.5} \subseteq \cdots \subseteq M_{\leq n-0.5} \subseteq M_{\leq n+0.5}=M
$$

such that, if $j \neq k, H^{k}\left(M_{\leq j+0.5}, M_{\leq j-0.5} ; \mathbb{Z}\right)=0$, and $H^{k}\left(M_{\leq k+0.5}, M_{\leq k-0.5} ; \mathbb{Z}\right)$ $\cong \mathbb{Z}^{\tau_{k}}$, where $\tau_{k}$ is equal to the number of points in $\Sigma f$ of index $k$. From this, by using the long exact sequence of a triple (as in Theorem 4.3), we obtain a differential complex whose cohomology is isomorphic to $H^{*}(M ; \mathbb{Z})$.

The following theorem is due to Smale. The proof involves successively modifying gradient-like vector fields.

Theorem 4.8.([41]) Every compact, smooth manifold admits a self-indexing Morse function.

The question, of course, is whether or not there is a useful stratified version of Theorem 4.8.

The first problem one encounters is how to define a well-behaved notion of "index" for a stratified Morse function. It turns out that such a notion of index exists in the complex setting, and so we will switch to that situation. Let $X$ be a smooth, complex algebraic variety, and let $\mathfrak{S}$ be a complex algebraic Whitney stratification of $X$. Let $n$ denote the complex dimension of $X$.

Definition 4.9. Suppose that $p$ is a stratified nondegenerate critical point of a smooth function $f: X \rightarrow \mathbb{R}$. Let $S$ denote the stratum containing $p$. Define the index of $f$ at $p$ by

$$
\operatorname{index}_{f}(p):=\operatorname{index}_{f_{\left.\right|_{S}}}(p)-\operatorname{dim}_{\mathbb{C}} S .
$$

If $f: X \rightarrow \mathbb{R}$ is a stratified Morse function, we say that $f$ is self-indexing provided that, at each stratified critical point $p$ of $f, \operatorname{index}_{f}(p)=f(p)$.

Note that the index of a critical point could be any integer between $-n$ and $n$, inclusive.

With this definition of self-indexing, what could one conclude from the existence of a self-indexing Morse function? As in the unstratified case, we would have a filtration

$$
\emptyset=X_{\leq-n-0.5} \subseteq X_{\leq-n+0.5} \subseteq X_{\leq-n+1.5} \subseteq \cdots \subseteq X_{\leq n-0.5} \subseteq X_{\leq n+0.5}=X .
$$

We wish to see what this tells us about the cohomology of $X$ with coefficients in a perverse sheaf $\mathbf{P}^{\bullet}$ (which is constructible with respect to $\mathfrak{S}$ ). Let us alter slightly our notation from Theorem 2.29; if $p \in X$, and $S$ is the connected component of the stratum containing $p$, then define the degree $k$ Morse module of $\mathbf{P}^{\bullet}$ at $p$ to be $m_{p}^{k}\left(\mathbf{P}^{\bullet}\right):=m_{S}^{k}\left(\mathbf{P}^{\bullet}\right)$. 
Then, according to Theorem 2.29 , for all $j \neq k, \mathbb{H}^{k}\left(X_{\leq j+0.5}, X_{\leq j-0.5} ; \mathbf{P}^{\bullet}\right)=0$, and

$$
B_{f}^{k}\left(\mathbf{P}^{\bullet}\right):=\mathbb{H}^{k}\left(X_{\leq k+0.5}, X_{\leq k-0.5} ; \mathbf{P}^{\bullet}\right) \cong \bigoplus_{\substack{p \in \Sigma f \\ \operatorname{index}_{f}(p)=k}} m_{p}^{0}\left(\mathbf{P}^{\bullet}\right)
$$

Once again using long exact sequences of triples, one obtains a differential complex

$$
0 \rightarrow B^{-n}\left(\mathbf{P}^{\bullet}\right) \stackrel{\partial^{-n}}{\longrightarrow} B^{-n+1}\left(\mathbf{P}^{\bullet}\right) \stackrel{\partial^{-n+1}}{\longrightarrow} \cdots \stackrel{\partial^{n-2}}{\longrightarrow} B^{n-1}\left(\mathbf{P}^{\bullet}\right) \stackrel{\partial^{n-1}}{\longrightarrow} B^{n}\left(\mathbf{P}^{\bullet}\right) \rightarrow 0,
$$

whose cohomology is isomorphic to $\mathbb{H}^{*}\left(X ; \mathbf{P}^{\bullet}\right)$.

This explains an important use of stratified self-indexing Morse functions, but do they exist? Grinberg's answer is "yes" if $X$ is complete (proper over $\mathbb{C}$ ); in particular, this includes the compact case.

Theorem 4.10.([17], 1.6) Every complete, nonsingular, Whitney stratified complex algebraic variety $(X, \mathfrak{S})$ admits a stratified self-indexing Morse function.

The proof of this result is again by successively altering gradient-like vector fields, but these vector fields must now be controlled along the strata.

\section{RESULTS ON THE MAIN THEOREM}

In his 1997 paper [22], and in [21], H. King gave a short proof of the Main Theorem of Stratified Morse Theory. In the first form of this result, King's hypotheses are weaker; he does not require the critical point to be nondepraved. His conclusion is correspondingly weaker; one does not know that one obtains standard local Morse data.

Let $M$ be a smooth manifold, and let $f: M \rightarrow \mathbb{R}$ be a smooth function. Let $X \subseteq M$ be a locally closed Whitney stratified set. Let $p \in X$, and let $S$ be the stratum containing $p$.

Theorem 4.11.([22], Theorem 5; [21], Theorem 9) Suppose that $d_{p} f$ is a nondegenerate conormal covector, and that $p$ is an isolated stratified critical point of $f$. Then, in a neighborhood of $p$, the product of Morse data for $f_{\left.\right|_{S}}$ and for $f_{\left.\right|_{X \cap D}}$, where $D$ is any submanifold of $M$ transverse to $S$ with $D \cap S=\{p\}$, is Morse data for $f$.

It is somewhat difficult in [22] to determine what King means by "Morse data"; this is clarified on page 3 of [21]. By "Morse data", King means local Morse data, $\left(C, C \cap f^{-1}(-\delta)\right)$, where $\delta>0$ is small and $C$ is a certain type of neighborhood of $p$ (part of the critical point data), which need not be a ball with respect to some Riemannian metric. 
However, in Lemma 12 of [21], under the assumption that $f$ has a nondepraved critical point at $p$, King does, in fact, recover the full content of the Main Theorem (Theorem 2.16) via a totally different proof than that of [16].

One final comment on King's papers: on page 8 of [22], and on page 10 of [21], King remarks that, in [16], Goresky and MacPherson care only about the intersection homology of the Morse data. As we saw and discussed in Section 3, it is true that Goresky and MacPherson were motivated to create Stratified Morse Theory to apply to intersection homology, but - as we also saw in Section 3 this was certainly not all that they were interested in in [16].

In addition to the work of King on Stratified Morse Theory on the level of homeomorphisms, there have been two large works dealing with results on the level of hypercohomology (or derived category) with coefficients in complexes of sheaves. These are the theories developed by Kashiwara and Schapira in Chapter V of [20], and Schürmann in Chapters 4 and 5 of [39]. In essence, these theories make rigorous and greatly expand upon the comment made in I.4.1 of [16]: spaces change as one moves in the direction of characteristic covectors. The microlocal approaches of Kashiwara, Schapira, and Schürmann proceed from a fundamental generalization of this precept: a complex of sheaves $\mathbf{F}^{\bullet}$ on a space $X$ determines "characteristic covectors with respect to $\mathbf{F}^{\bullet}$ ", i.e., the directions in which the hypercohomology of $X$ with coefficients in $\mathbf{F}^{\bullet}$ will change. This collection of characteristic covectors with respect to $\mathbf{F}^{\bullet}$ is called the micro-support of $\mathbf{F}^{\bullet}$, denoted by $S S\left(\mathbf{F}^{\bullet}\right)$.

The results of Kashiwara and Schapira in Chapter V of [20] provide powerful tools for studying hypercohomology of real or complex spaces, but one does not see a precise correlation with the results of [16]. On the other hand, in Chapters 4 and 5 of [39], Schürmann essentially redefines all of the concepts, and reproves all of the results, of Stratified Morse Theory, but always with respect to a complex of sheaves. This allows him to optimize his hypotheses for derived category results, and allows him to simplify a number of proofs. For instance, Schürmann's derived category version of the Main Theorem of Stratified Morse Theory appears in Theorem 5.3.3 of [39], and its proof is considerably less difficult than that of Theorem 2.16.

While it would take us too far afield to provide the requisite background to explain results from [20] or [39], we can provide one of our own applications of the micro-local approach. This is a generalization of Theorem 2.31; it is a complex, hypercohomological version of the Main Theorem. First, we need a definition, and we would like to characterize, using Stratified Morse Theory data, the micro-support in the complex analytic setting. 
Suppose that $X$ is a complex analytic space, embedded in a complex manifold $M$. Let $\tilde{f}: M \rightarrow \mathbb{C}$ be complex analytic, and let $f:=\tilde{f}_{\left.\right|_{X}}$. Let $\mathbf{F}^{\bullet}$ be a bounded complex of sheaves of $R$-modules on $X$, constructible with respect to a complex analytic Whitney stratification $\mathfrak{S}$, with connected strata.

Definition 4.12. A stratum $S \in \mathfrak{S}$ is $\mathbf{F}^{\bullet}$-visible if and only if it possesses a non-zero Morse module $m_{S}^{k}\left(\mathbf{F}^{\bullet}\right)$ in some degree $k$, i.e., $\mathbb{H}^{*}\left(\mathbb{N}_{S}, \mathbb{L}_{S} ; \mathbf{F}^{\bullet}\right) \neq 0$.

Theorem 4.13.([30], 4.13) The micro-support, $S S\left(\mathbf{F}^{\bullet}\right)$, of $\mathbf{F}^{\bullet}$ is equal to

$$
\bigcup_{\mathbf{F} \bullet-\text { visible } S} \overline{T_{S}^{*} M}
$$

Our generalization of Theorem 2.31 is:

Theorem 4.14.([30], 3.4 and 5.3) A point $p$ is an isolated point in the support of $\phi_{f}[-1] \mathbf{F}^{\bullet}$ if and only if $p$ is an isolated point in

$$
\left\{x \in X \mid\left(x, d_{x} \tilde{f}\right) \in \bigcup_{\mathbf{F}^{\bullet} \text {-visible } S} \overline{T_{S}^{*} M}\right\}
$$

and, when these equivalent condition hold, for all $k$, there is an isomorphism

$$
H^{k}\left(\phi_{f} \mathbf{F}^{\bullet}[-1]\right)_{p} \cong \bigoplus_{\mathbf{F}^{\bullet} \text {-visible } S}\left(m_{S}^{k}\left(\mathbf{F}^{\bullet}\right) \otimes R^{\mu_{S}}\right),
$$

where $\mu_{S}$ is the number of complex nondegenerate critical points of a small, complex perturbation of $f$ which occur near $p$ on the stratum $S$. Thus, $\mu_{S}$ equals the intersection number $\left(\overline{T_{S}^{*} M} \cdot \operatorname{im} d \tilde{f}\right)_{\left(p, d_{p} \tilde{f}\right)}$.

Remark 4.15. On the level of Euler characteristics, this is the index theorem proved independently by Ginsburg in [11], Lê in [26], and Sabbah in [38]. In the proof of Theorem 4.14, we used many results from Chapter V of [20]. However, one can prove this theorem very quickly by using enriched cycles (see [32]) and "enriching" Lê's proof of the Euler characteristic result.

The reader should note that the hypothesis that $p$ is an isolated point of the support of $\phi_{f}[-1] \mathbf{F}^{\bullet}$ is strictly weaker than requiring that $f$ has a stratified isolated critical point at $p$, i.e., that $p$ is an isolated point of $\Sigma_{\mathfrak{S} f}$. In Theorem 4.14, $f$ may have non-isolated critical points on strata which are not $\mathbf{F}^{\bullet}$-visible.

If, in fact, $p$ is an isolated point of $\Sigma_{\mathfrak{S}} f$, then one can simply perturb $f$ slightly, in a complex analytic manner, to obtain a function with only complex nondegenerate critical points (as in Theorem 2.31) in a neighborhood of $p$, and the conclusion of Theorem 4.14 follows easily from Theorem 2.31. This result, with constant coefficients, is due to Siersma ([40]) and Tibăr ([42]), and we proved it with coefficients in $\mathbf{F}^{\bullet}$ in Theorem 3.2 of [29]. A proof along the same lines 
can be made to work under the weaker hypothesis that $p$ is an isolated point of the support of $\phi_{f}[-1] \mathbf{F}^{\bullet}$; however, it is complicated to deal with the possibly non-isolated critical points of $f$ on $\mathbf{F}^{\bullet}$-invisible strata, and prove that they do not matter when applying Stratified Morse Theory to the boundary strata of a compact neighborhood of $p$.

If $p$ is not an isolated point in the support of $\phi_{f}[-1] \mathbf{F}^{\bullet}$, then a result for the stalk cohomology, like that in Theorem 4.14, is not known. However, one can calculate the Morse modules of the strata of any stratification with respect to which $\phi_{f}[-1] \mathbf{F}^{\bullet}$ is constructible. This generalization of Theorem 4.14 appears in Theorem 3.4 of [32].

\section{RECTIFIED HOMOTOPICAL AND HOMOLOGICAL DEPTH}

In [18], Hamm and Lê prove a number of conjectures made by Grothendieck on homotopy (or homology) relations between complex algebraic varieties and their hyperplane sections (or more general subvarieties). These results are similar in flavor to those appearing in [16], II.1 and to the homotopy results we discussed in Section 3. Most of the proofs in [18] do not use Stratified Morse Theory. While the authors were familiar with Stratified Morse Theory, and mention in the introduction that they could have used this theory in some of their proofs, they wished to write their paper using techniques that were known to Grothendieck.

Nonetheless, in the proofs of Lemma 3.3.3, Lemma 3.4.2, and Theorem 4.2.1, Hamm and Lê are "forced" to use Stratified Morse Theory. In addition, in Theorem 4.1.2 of [18], Hamm and Lê characterize rectified homotopical depth in terms of the normal Morse data of strata. Below, we define rectified homotopical and homological depth, and give Theorems 4.1.2 and 4.2.1 of [18].

Let $X$ be a complex analytic space, with a complex analytic Whitney stratification $\mathfrak{S}$, with connected strata. Let $X_{i}$ be the union of strata of dimension at most $i$. Fix a base ring $R$, which is either $\mathbb{Z}$ or $\mathbb{Q}$.

Definition 4.16.([18], 1.1) The rectified homotopical (resp., homological) depth, $\operatorname{rhd}_{\mathfrak{S}}(X)$ (resp., $\operatorname{rHd}_{\mathfrak{S}}(X ; R)$ ), of $X$ is greater than or equal to $n$ if and only if, for all $i$, for all $x \in X_{i}-X_{i-1}$, there exists a fundamental system $\left\{\mathcal{U}_{\alpha}\right\}_{\alpha}$ of neighborhoods of $x$ in $X$ such that, for all $\alpha$, the pair $\left(\mathcal{U}_{\alpha}, \mathcal{U}_{\alpha}-X_{i}\right)$ is $(n-1-i)$ connected (resp., $H_{k}\left(\mathcal{U}_{\alpha}, \mathcal{U}_{\alpha}-X_{i} ; R\right)=0$ for all $\left.k<n-i\right)$.

Of course, this completely determines the numbers $\operatorname{rhd}_{\mathfrak{S}}(X)$ and $\operatorname{rHd}_{\mathfrak{S}}(X ; R)$.

In Lemma 1.3, Hamm and Lê prove that $\operatorname{rhd}_{\mathfrak{S}}(X)$ and $\operatorname{rHd}_{\mathfrak{S}}(X ; R)$ are independent of the Whitney stratification $\mathfrak{S}$, and thus drop the reference to the stratification from the terminology and notation. One writes simply $\operatorname{rhd}(X)$ and $\operatorname{rHd}(X ; R)$. 
The rectified homotopical depth can be characterized nicely in terms of $\left(\mathbb{N}_{S}, \mathbb{L}_{S}\right)$.

Theorem 4.17.([18], 4.1.2) The following conditions are equivalent:

(1) $\operatorname{rhd}(X) \geq n$;

(2) for all $S \in \mathfrak{S}$, the pair $\left(\mathbb{N}_{S}, \mathbb{L}_{S}\right)$ is $(n-\operatorname{dim} S-1)$-connected.

Hamm and Lê prove the following result by considering the Stratified Morse Theory of a small perturbation of the function given by negative the distance squared to $p$.

Theorem 4.18.([18], 4.2.1) Assume that $X$ is embedded in a complex manifold, endowed with a Riemannian metric. Let $Z$ be a closed complex analytic subspace of $X$, and let $p \in Z$. Suppose that $\operatorname{rHd}(X ; \mathbb{Z}) \geq n$. Let $g:(\mathcal{U}, p) \rightarrow(\mathbb{C}, 0)$ be a complex analytic function defined on a neighborhood $\mathcal{U}$ of $p$ in $X$.

Then, for $0<\alpha \ll \epsilon \ll 1$, for all $t \in \mathbb{C}$ such that $|t|=\alpha$, the pair of spaces

$$
\left(B_{\epsilon}(p) \cap(X-Z),\left[\left(S_{\epsilon}(p) \cap g^{-1}\left(\mathbb{D}_{\alpha}\right)\right) \cup\left(B_{\epsilon}(p) \cap g^{-1}(t)\right)\right] \cap(X-Z)\right)
$$

is $(n-1)$-connected, where $\mathbb{D}_{\alpha} \subseteq \mathbb{C}$ is the closed disk of radius $\alpha$ centered at 0 .

\section{DISCRETE MORSE THEORY}

In [9] and [8], R. Forman develops a discrete, combinatorial version of Morse Theory and Stratified Morse Theory. We present the fundamental definitions and main theorem here. We follow the exposition in [10].

First, we recall some basic facts on abstract simplicial complexes.

Definition 4.19. An abstract finite simplicial complex consists of a finite set $V$ of vertices, together with a set $K$ of non-empty subsets of $V$ such that, for all $v \in V,\{v\} \in K$ (by abuse, one frequently writes $V \subseteq K$ ), and, if $\alpha \in K$ and $\emptyset \neq \beta \subseteq \alpha$, then $\beta \in K$.

The elements of $K$ are called simplices, and the dimension, $\operatorname{dim} \alpha$, of a simplex $\alpha \in K$ is defined to be the cardinality of $\alpha$ minus 1 , i.e., $\operatorname{dim} \alpha=(\# \alpha)-1$. To indicate that $\alpha$ has dimension $p$, we write $\alpha^{(p)}$.

If $\alpha, \beta \in K$, we write $\alpha<\beta$ to indicate that $\alpha$ is a proper subset of $\beta$.

A discrete Morse function on the abstract simplicial complex $(V, K)$ is a function which essentially assigns higher numbers to higher-dimensional simplices, with at most one exception, locally, at each simplex.

Definition 4.20. A function $r: K \rightarrow \mathbb{R}$ is a discrete Morse function on $(V, K)$ if and only if, for all $\alpha^{(p)} \in K$,

$$
1 \geq \#\left\{\beta^{(p+1)}>\alpha^{(p)} \mid r(\beta) \leq r(\alpha)\right\}
$$


and

$$
1 \geq \#\left\{\gamma^{(p-1)}<\alpha^{(p)} \mid r(\gamma) \geq r(\alpha)\right\} .
$$

Suppose now that $r$ is a discrete Morse function on an abstract finite simplicial complex $(V, K)$. We need the analog of a "critical point".

Definition 4.21. A simplex $\alpha^{(p)}$ is a critical simplex of $r$ if and only if

$$
0=\#\left\{\beta^{(p+1)}>\alpha^{(p)} \mid r(\beta) \leq r(\alpha)\right\}
$$

and

$$
0=\#\left\{\gamma^{(p-1)}<\alpha^{(p)} \mid r(\gamma) \geq r(\alpha)\right\}
$$

Now, suppose that we have a topological space $X$, together with a (geometric) finite simplicial decomposition. One frequently expects to be able to find a $\mathrm{CW}$ decomposition of $X$ which has fewer cells than the original simplicial decomposition. This is addressed by the main theorem of discrete Morse theory.

Theorem 4.22. ([10], 2.5) Suppose that $(V, K)$ an abstract finite simplicial complex with a discrete Morse function. Then, the geometric realization $|K|$ is homotopy-equivalent to a $C W$ complex with exactly one cell of dimension $p$ for each critical simplex of dimension $p$.

An example will aid the reader in understanding the above definitions and theorem, and will help explain in what sense this yields of version of "Stratified Morse Theory".

Example 4.23. Consider the singular space $X$ consisting of two circles joined by a line segment. Below, we have drawn the space, together with a simplicial subdivision with vertices $\{a, b, c, d, e, f\}$. This simplicial complex has 6 zerodimensional simplices, and 7 one-dimensional simplices.

The numbers that we have drawn on the outside of the diagram are the values of a discrete Morse function $r$. For instance, $r(\{b\})=2$ and $r(\{a, b\})=3$.

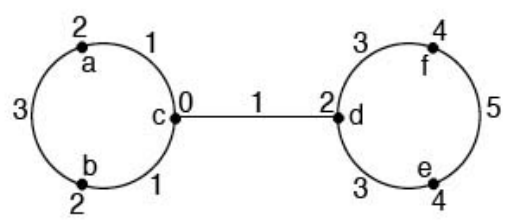

The reader should verify that this function does, in fact, satisfy the conditions for a discrete Morse function, and that the critical simplices are precisely $\{a, b\}$, $\{c\}$, and $\{e, f\}$. Thus, $X$ is homotopy-equivalent to a CW complex with one 0cell and two 1-cells. Of course, this is obvious in this example, since $X$ is clearly homotopy-equivalent to a figure eight. 
There is a connection with smooth functions on stratified spaces. Sometimes one can "smooth out" a discrete Morse function to obtain a smooth one, or one can "discretize" a smooth (stratified) Morse function to obtain a discrete one. For instance, the discrete Morse function $r$ above was obtained by discretizing the function given by (a small perturbation of) the distance (or distance squared) from the vertex $c$.

\section{REFERENCES}

[1] Beilinson, A. A., Bernstein, J., Deligne, P. Faisceaux pervers, volume 100 of Astérisque. Soc. Math. France, 1981.

[2] Björner, A. and Ekedahl, T. Subspace arrangements over finite fields: cohomological and enumerative aspects. Adv. Math., 129:159-187, 1997.

[3] Björner, A. and Ziegler, G. Combinatorial stratification of complex arrangements. Jour. AMS, 5:105-149, 1992.

[4] Cohen, D. Cohomology and Intersection Cohomology of Complex Hyperplane Arrangements. PhD thesis, Northeastern University, 1992.

[5] Cohen, D. and Orlik, P. Stratified Morse Theory in Arrangements. Pure and Applied Math. Quarterly. Special Issue: In Honor of Robert D. MacPherson, Part 1 of 3, vol. 2, no. 3:673-697, 2006.

[6] Dimca, A. Sheaves in Topology. Universitext. Springer-Verlag, 2004.

[7] Feichtner, E. M. and Ziegler, G. On cohomology algebras of complex subspace arrangements. Trans. AMS, 352:3523-3555, 2000.

[8] Forman, R. A Discrete Morse Theory for Cell Complexes. In S. T. Yau, editor, Geometry, Topology \& Physics for Raoul Bott, pages 112-125. International Press, 1995.

[9] Forman, R. Morse Theory for Cell Complexes. Adv. Math., 134:90-145, 1998.

[10] Forman, R. A User's Guide to Discrete Morse Theory. Séminaire Lotharingien de Combinatoire, 48:B48c, 2002.

[11] Ginsburg, V. Characteristic Varieties and Vanishing Cycles. Invent. Math., 84:327-403, 1986.

[12] Goresky, M. and MacPherson, R. Intersection Homology Theory. Topology, 19:135-162, 1980.

[13] Goresky, M. and MacPherson, R. Intersection Homology II. Invent. Math., 71:77-129, 1983.

[14] Goresky, M. and MacPherson, R. Morse Theory and Intersection Homology, volume 101 of Astérisque, pages 135-192. Soc. Math. France, 1983.

[15] Goresky, M. and MacPherson, R. Stratified Morse Theory, volume 40 of Proc. Symp. Pure Math., pages 517-533. AMS, 1983.

[16] Goresky, M. and MacPherson, R. Stratified Morse Theory, volume 14 of Ergeb. der Math. Springer-Verlag, 1988.

[17] Grinberg, M. Gradient-like flows and self-indexing in stratified Morse theory. Topology, 44:175-202, 2005.

[18] Hamm, H. and Lê, D. T. . Rectified homotopical depth and Grothendieck conjectures, volume 87 of Progr. Math., pages 311-351. Birkhäuser, 1990.

[19] Kashiwara, M. and Schapira, P. Microlocal Study of Sheaves, volume 128 of Astérisque. Soc. Math. France, 1985.

[20] Kashiwara, M. and Schapira, P. Sheaves on Manifolds, volume 292 of Grund. math. Wissen. Springer-Verlag, 1990.

[21] King, H. Sums of Isolated Critical Points. preprint. 
[22] King, H. Topology of Isolated Critical Points of Functions on Singular Spaces, volume 55 of Collection Travaux en Cours, pages 63-72. Hermann, 1997.

[23] Kirwan, F. An introduction to intersection homology theory, volume 187 of Pitman Research Notes in Math. Longman Scientific \& Tech., 1988.

[24] Kleiman, S. The Development of Intersection Homology Theory. In Peter L. Duren, editor, A century of mathematics in America, Part II, pages 543-585. AMS, 1987.

[25] Lazzeri, F. Morse Theory on Singular Spaces, volume 7-8 of Astérisque, pages 263-268. Soc. Math. France, 1973.

[26] Lê, D. T. Le concept de singularité isolée de fonction analytique. Advanced Studies in Pure Math., 8:215-227, 1986.

[27] Lê, D. T. and Teissier, B. Variétés polaires locales et classes de Chern des variétés singulières. Annals of Math., 114:457-491, 1981.

[28] Massey, D. Numerical Invariants of Perverse Sheaves. Duke Math. J., 73(2):307-370, 1994.

[29] Massey, D. Hypercohomology of Milnor Fibres. Topology, 35:969-1003, 1996.

[30] Massey, D. A Little Microlocal Morse Theory. Math. Ann., 321:275-294, 2001.

[31] Massey, D. Numerical Control over Complex Analytic Singularities, volume 778 of Memoirs of the AMS. AMS, 2003.

[32] Massey, D. Singularities and Enriched Cycles. Pacific J. Math., 215, no. 1:35-84, 2004.

[33] Mather, J. Notes on Topological Stability. Notes from Harvard Univ., 1970.

[34] Milnor, J. Morse Theory, volume 51 of Annals of Math. Studies. Princeton Univ. Press, 1963. Based on lecture notes by Spivak, M. and Wells, R.

[35] Orlik, P. and Solomon, L. Combinatorics and Topology of Complements of Hyperplanes. Invent. Math., 56:167-189, 1980.

[36] Orlik, P. and Terao, H. Arrangements of Hyperplanes, volume 300 of Grund. Math. Wissen. Springer-Verlag, 1992.

[37] Pignoni, R. Density and Stability of Morse Functions on a Stratified Space. Ann. Scuola Nor. Sup. Pisa, series IV, 6:593-608, 1979.

[38] Sabbah, C. Quelques remarques sur la géométrie des espaces conormaux. Astérisque, 130:161-192, 1985.

[39] Schürmann. Topology of Singular Spaces and Constructible Sheaves, volume 63 of Monografie Matematyczne. Birkhäuser, 2004.

[40] Siersma, D. A bouquet theorem for the Milnor fibre. Joun. Alg. Geom., 4:51-66, 1995.

[41] Smale, S. On gradient dynamical systems. Ann. of Math. (2), 74:199-206, 1961.

[42] Tibăr, M. Bouquet Decomposition of the Milnor Fiber. Topology, 35:227-241, 1996.

[43] Zaslavsky, T. Facing up to arrangements: face count formulas for partitions of space by hyperplanes, volume 154 of Memoirs of the AMS. AMS, 1975.

[44] Ziegler, G. and Živaljević, R. Homotopy types of subspace arrangements via diagrams of spaces. Math. Ann., 295:527-548, 1993.

David B. Massey

Department of Mathematics, 567 Lake Hall, Northeastern University, Boston, MA 02115, USA

E-mail: dmassey@neu.edu 